\title{
Optimal Buffer Allocation in Multi-Product Repairable Production Lines Based on Multi-State Reliability and Structural Complexity
}

\author{
Jianguo Duan ${ }^{1, *}$, Nan $\mathrm{Xie}^{2}$ and Lianhui $\mathrm{Li}^{3}$ \\ ${ }^{1}$ China Institute of FTZ Supply Chain, Shanghai Maritime University, Shanghai 201306, China \\ [e-mail: caleb_duan@aliyun.com] \\ ${ }^{2}$ Sino-German College of Applied Sciences, Tongji University, Shanghai 200092, China \\ [e-mail: xienan115@tongji.edu.cn] \\ ${ }^{3}$ Ningxia Key Laboratory of Intelligent Information and Big Data Processing, North Minzu University, Yinchuan \\ 750021, China \\ [e-mail: lilianhui@nmu.edu.cn] \\ *Corresponding author: Jianguo Duan
}

Received October 14, 2019; revised January 9, 2020; accepted February 9, 2020;

published April 30, 2020

\begin{abstract}
In the design of production system, buffer capacity allocation is a major step. Through polymorphism analysis of production capacity and production capability, this paper investigates a buffer allocation optimization problem aiming at the multi-stage production line including unreliable machines, which is concerned with maximizing the system theoretical production rate and minimizing the system state entropy for a certain amount of buffers simultaneously. Stochastic process analysis is employed to establish Markov models for repairable modular machines. Considering the complex structure, an improved vector UGF (Universal Generating Function) technique and composition operators are introduced to construct the system model. Then the measures to assess the system's multi-state reliability and structural complexity are given. Based on system theoretical production rate and system state entropy, mathematical model for buffer capacity optimization is built and optimized by a specific genetic algorithm. The feasibility and effectiveness of the proposed method is verified by an application of an engine head production line.
\end{abstract}

Keywords: Multi-state reliability; structural complexity; repairable production system; buffer allocation

This research was funded by National Natural Science Foundation of China (No. 71471139), and Intelligent Manufacturing Comprehensive Standardization and New Mode Application Projects of China, The Third Batch of Ningxia Youth Talents Supporting Program (No.TJGC2018048) and University-enterprise Joint Project of North Minzu University (No. 2018HLZ07). 


\section{Introduction}

\subsection{Research motivation}

$\mathbf{F}_{\text {or a production system, there are various uncertainties that will seriously affect the normal }}$ operation of themselves [1]. Although flexibility of a production system itself sometimes compensates for some certain uncertainties, it has a limited effect. In most cases, the relationships between production uncertainties and flexibility are balanced by arranging a certain number of buffers between workstations [2]. The problem that how to make the buffer stations have enough space to store the WIP (Work in Process), and minimize the equipment cost and maintenance cost brought by the intermediate buffers, while avoiding time waste and logistics interruption due to excessive travel of WIP in intermediate buffers, and maintaining the reliable, controllable and stable operations has always been a key issue in the design of production systems [3-10]. Among them, system modeling and analysis are the basis for the buffer capacity distribution problem, and the optimization method is the means to obtain reasonable allocation solutions $[11,12]$. Therefore, based on the analysis of multi-state reliability and structural complexity, it is of great significance to study the allocation planning method and optimization algorithm of buffer capacity for production system design.

\subsection{Literature review}

\subsubsection{System reliability research}

Reliability is an extremely important indicator for performance measure of production systems. The complexity of reliability depends on the rather complex relationship between all machine and equipment events that happen asynchronously on discrete time. As well as machine failures, we also need to consider machine congestion, lack of materials and reduced operation [13]. Therefore, with the continuous expansion of the scale of production system and the complexity of the structure, reliability becomes more and more important $[14,15]$.

The reliability model is a mathematical model used to describe the logical relationships of failures between production systems and equipment, and it is an essential part of reliability assessment. In recent years, with the rapid development of reliability modeling technology, the reliability modeling methods for complex systems have also matured [16-20]. In binary reliability structure, there are only two states for a component: complete performance and complete failure, that is, only "yes" and "no" binary values are used to describe whether a system can perform the specified functions. However, in a production system, the system or equipment tends to present multiple states which are distributed from optimal operation to complete failure. In different states, a system or equipment has different performance levels, that is, both the system and the equipment can be called multi-state system [21]. The reliability analysis theory provides a new train of thought for the research of multi-state production system [22, 23]. Unlike the traditional analysis methods, the recently proposed UGF is more time-saving and more efficient. It is an important approach in modern discrete mathematics, which allows algebraic methods to replace combination operations to calculate the performance distributions of multi-state systems. So this approach is a powerful tool for large-scale multi-state production system reliability modeling [24-27]. 


\subsubsection{System complexity research}

In the study of advanced production systems, system complexity is a very prominent problem. Traditionally, complexity can be classified into two types, namely static complexity which is also called structural complexity and dynamic complexity which is also called operational complexity. As an inherent characteristic of engineered system, structural complexity is time-independent. As a characteristic concerned with the operational behaviors of engineered system, dynamic complexity is time-dependent [28]. How to survey the complexity of production systems and effectively control and manage it has important practical significance for the design, analysis and operation control of production systems. It has also become one of the hot issues in the field of advanced manufacturing in recent years $[29,30]$. The structural complexity of a production system is the amount of information needed to describe the states that each production resource under static states is expected to have, while information entropy is an effective way to quantitatively evaluate the uncertainties of a production system.

Manufacturing Systems Group at Oxford University first applied information theory to complexity assessment of production systems. Since then, Frizelle et al. proposed two kinds of models to measure the complexity of production systems after analyzing their dynamic queuing behaviors [31, 32]. Efstathiou et al. demonstrated that the system entropy is equal to the complexity of the production system and divided it into three categories, namely structural complexity, dynamic complexity and decision-making complexity [33]. EIMaraghy et al. used the information entropy to evaluate the production systems' complexity by analyzing the main elements such as machining equipment, intermediate buffers, logistics equipment, and their interconnection and interdependencies [34-37]. Modeling and optimizing of model assembly line system with serial, parallel and hybrid configurations are studied by $\mathrm{Hu}$ et al. These configurations can be applied to product assembly sequence planning and configuration selection of assembly systems [38-41]. Some other scholars have also used different methods to measure the complexity of production systems from different perspectives such as operational complexity [42] or dynamic complexity [43].

Most of the literatures on production system complexity just referred to individual resources. Almost modeling methods are based on the assumption of state independence and the system complexity is usually obtained by simple superposition of resource complexity. But in fact, our concern is the complexity of the entire system rather than that of individual resources. Therefore, modeling and measuring the production system complexity in the mass not only helps us understand and grasp the uncertainty on system level, but also seems more reasonable. In addition, system resources are related and interact with each other, in fact, the assumption that mentioned above is not always true, and sometimes there will be some coupling between resource states. For example, in machining production lines, the buffer states will affect the machining equipment. Therefore, the complexity of the entire system is not a simple superposition of the amount of information on each resource. Furthermore, almost existing studies focus on theoretical research and ignores the applications in practice. How to apply complexity theory to production practice has become an important issue.

\subsubsection{Buffer allocation research}

The buffer capacity allocation problem of production systems mainly includes two categories: one is to solve the minimum total capacity when the system productivity is 
determined, and the other is to maximize the system productivity by optimal allocation when the total buffer capacity is determined [44]. It is important and complex in production line designing. As a result, it has been studied for more than fifty years with lots of research publications in the literature.

To guarantee the expected production rate in unreliable machine serial line, Enginarlar et al. researched the lowest buffering level [3]. Oriented for the buffered production line, Konishi researched blocking and starving under the background of nonlinear dynamics and put forward a strategy to avoid blocking and starving based on frequency response and Ho-norm [5]. By blancing the shortage cost, holding cost, repair cost for warranty, rework cost, material cost, labour/energy costs and cost for maintenance, Sankar solved the optimum buffer level problem and production run time problem [7]. To maximize the average system production rate and minimize the total buffer size, Kose et al. proposed a simulation optimization method based on hybrid evolutionary algorithm and optimized the above two conflicting objectives [8]. Sabry and Dmitriev studied the assembly line function having the characteristic of reliable, unpaced merging and asymmetric buffer storage sizes [10]. Oriented for mutiple key performance measures of production systems, Shaaban et al. put forward a MOO method to search and analyze the supreme patterns of buffer allocation and workload imbalance [12]. Aiming at the optimization of unreliable and imperfect production system, Radhoui et al. developed an integrated model based on quality control, preventive maintenance and buffer allocation sizing [45]. Walid et al. explored the influence of buffer allocation size on decreasing the impact of production line blocking and starvation, tested the performance indicators and solved the throughput problem of an automatic multi-product production line [46]. To optimize the production system with unreliable machines and inter-operational buffers, Matta et al. proposed an output function Kriging approximation-based sequential search method with limited capacity approximated by analytical models [47]. Papadopoulos et al. experimentally studied a total of 105 different serial production line buffer allocation problem using five different search methods including tabu search, genetic, simulated annealing, myopic and complete enumeration to record the throughput achieved and CPU time required [48]. By extending the existing column generation algorithm, Alfieri et al proposed a row-column generation algorithm to solve the approximated buffer allocation problem in transfer and assembly/disassembly multi-stage production systems [49]. Renna investigated the buffer allocation problem with preventive maintenance policy, and presented a dynamic control of the buffers' level and the interval between two consecutive preventive actions [50]. Motlagh et al address a novel problem considering two sets of decision variables and three objective functions in actual unreliable production lines, and developed an optimization method based on Design of Experiments and Response Surface Methodology [51]. These scholars have only studied the buffer allocation problem from a certain aspect.

However, apart from them, there are several reviews by Park [52], Gershwin [44], Demir [53]. In addition, Weiss et al. took the most comprehensive survey on the current technical level about buffer allocation problem in production lines. In this article, they classified and reviewed the literature on buffer allocation problem about different types of the optimizing problem, the application approaches and the consideration of actual or common cases [54].

Based on the above literature review, the following limitations can be drawn:

- Non-serial multi-stage manufacturing systems are widely employed in industrial practices. However, the past studies on buffer allocation problems used to works on 
transfer lines with identical jobs entering the system rather than non-serial multi-stage production lines which focuses on the diversity of production capability and the size of production capacity simultaneously. In the multi-product repairable production lines, elements (manufacturing machines, material handling equipment, etc.) and system may be in different states, from normal operation to complete failure, related to product diversity and production rate within a prescribed period due to the polymorphism of machines, system configurations, and intermediate buffers. It is a unique repairable multi-state manufacturing system with finite buffer stations for production of a product family.

- Most of the researches on unreliable buffer allocation are focused on single objective function, and their popular objective functions considered in the literature are throughput (production rate) maximization and total cost minimization. As the scales and flexibilities of production lines expand and their structure become more complex, it is increasingly important to make them more productive and predictive simultaneously with multiple contradictory objectives of maximizing system multi-state reliability and minimizing system structural complexity. But actually, all related literature surprisingly lacks the consideration of them.

- Although simulation method is one of the popular evaluation methods for unreliable buffer allocation problems and it can deal with real-world systems with large scale problems, probabilistic parameters, uncertainties, etc., it is essentially an approximate method which can only provide an estimation for the actual throughput rate. In some cases, the approximation level of the method can have a negative impact on the solutions, and the time complexity can be relatively large.

- For buffer allocation problems, modeling is the first and crucial step for searching the optimal solutions. So in both reliability and complexity evaluations of the manufacturing systems, we must obtain all the final states accurately by an appropriate multi-state modelling method. Some traditional techniques such as Bayesian network, Petri net, Binary decision diagram, etc. don't work for multi-state manufacturing systems due to great number of system states.

- The recently emerged Universal generating function technique has been proved to be effective for the reliability evaluation of multi-state manufacturing system without buffer stations, but were hardly used in the evaluation of system complexity nor in solving buffer allocation problems of multi-state manufacturing system with finite buffer stations integrated with stochastic process and generic algorithm. below:

The proposed research in this paper overcomes the above limitations in the respect of

- In order to describe the diversity of production capability and the size of production capacity simultaneously in one function, we propose an improved vector Universal Generating Function and two composition operators by extending the dimensions of traditional Universal Generating Function. Hence, the reliability of non-serial multi-state repairable production line with finite buffers is measured through analysis of the effects of the buffers' states on the machines' states and decomposition of the system configuration. By replacing combinational operation with the algebraic operation, the problem of combination explosion during reliability modeling with stochastic process method is eliminated.

- For the sake of cover the shortage that traditional complexity models are based on the assumption that all states are mutually independent, structural complexity 
model of multi-state manufacturing system with finite buffers using state entropy is introduced combining with the improved vector Universal generating function. This technique can evaluate the system structural complexity precisely from the system level meanwhile overcome the shortcomings of the existing methods with the assumption of state independence and ensure the accurate and quantitative evaluation of system state uncertainties.

- Taking system multi-state reliability and system structural complexity as the optimization objective functions, buffer stations of the production line are optimally configured with the tools of extended vector Universal generating function and Genetic algorithm. This method can formulate the buffer allocation problems as a function of the system structure and the performance distributions of its elements, and discover the optimal composition of all the factors influencing the entire system performance subject to specific constraints efficiently. The optimization solutions gives some practical proposals for the designing and planning of multi-product repairable production lines with multiple stages and non-serial configurations, such as engine-head machining lines.

\subsection{Outline}

Our paper's rest is organized as follows. In Section 2, we describe the multi-product reparable production system and propose 6 assumptions as the basis of following study. In Section 3, we give all the details of our machine modeling method. The full calculation procedures for the vector UGF of the entire system and their composition operators are defined in Section 4. In addition, we also give two indicators, multi-state reliability and structural complexity, as well as their measures in this section. In Section 5, optimal allocation method of buffer capacity including the problem formulation, the optimization approach and equality constraints are illustrated. Then, case study is followed in Section 6 and conclusion is followed in Section 7.

\section{Statement of the problem and several assumptions}

It is supposed that there is a multi-state production line which is repairable and serial-parallel. There are $m$ unreliable workstations and $m-1$ intermediate buffer for part family $\boldsymbol{P}=\left\{P_{1}, P_{2}, \cdots, P_{K}\right\}$. For each workstation, all machines are the same, but the number of machines may be different. Between each two workstations of the production line, there are several buffer stations. We suppose that the $j$ th machine in workstation $i$ is represented as $M_{i}$, and the performance level number is $m_{i j}$, which can be expressed by vector $\boldsymbol{g}_{i j}=\left\{\boldsymbol{g}_{i j 1}, \cdots, \boldsymbol{g}_{i j m_{j}}\right\}$, here the performance level of $M_{i j}$ in state $k$ is denoted by $g_{i j k}$ with $k=1, \ldots, m_{i j}$. We use $g_{i j 1}$ to express the worst performance state, while $\boldsymbol{g}_{i j m_{j j}}$ is the best performance state. In multi-state production lines, the best performance state means that the machine is working at the highest speed for each part of the part family, and the worst performance state indicates that the machine is in complete failure and therefor its production rate for each part is zero.

$\boldsymbol{G}_{i j}(t)$ is the performance rate. When $t \geq 0, \boldsymbol{G}_{i j}(t)$ is a random variable and $\boldsymbol{G}_{i j}(t) \in \boldsymbol{g}_{i j}$. $\boldsymbol{G}_{i j}(t)$ corresponds to the state and probability:

$$
X_{i j}(t) \in \boldsymbol{x}_{i j}=\left\{1, \cdots, m_{i j}\right\}, \boldsymbol{p}_{i j}=\left\{p_{i j 1}, p_{i j 2}, \cdots, p_{i j m_{i j}}\right\}
$$


The total number of combinations of system states corresponding to all machine states is:

$$
K=\prod_{i=1}^{m}\left(\prod_{j=1}^{n_{i}} m_{i j}\right)
$$

where $n_{i}$ is the number of machines in workstation $i$.

For the sake of assessing the comprehensive system performance distribution in detail, it is necessary to clarify all possible performance and corresponding state probabilities. The aim is to describe multiple attributes more clearly. Based on the following assumptions, this paper studies the reliability modeling of repairable production line with finite buffer stations.

(1) The research object is a non-serial repairable production line. When the machines in the system are broken down, they can be repaired in time and can completely restore normal working performance.

One of the most important properties of the exponential distribution is memoryless property, that is, the probability of an event occurring over a period of time has nothing to do with the elapsed time when no event has occurred. In many practical situations, this property is very realistic. This is why exponential distributions are widely used to model processing time, failure time, and repair time. Here, we assume that the processing time, mean time between failures and repair time of each machine utilized in this paper follow the exponential distributions of parameters $\omega_{i j}$ (production rate), $\lambda_{i j}$ (failure rate) and $\mu_{i j}$ (repair rate), respectively.

(2) With regard to all part types in the part family, finite capacity of each buffer station remains unchanged and no fault exists in the transfer of the workpiece to the buffer station.

(3) Matching with the production rate, the time to process the workpiece stored in or taken out of the buffer is very short. As a result, we usually neglect it.

(4) For all the machines located in the first process, they have enough workpieces to process. So the idle phenomenon due to material shortage will not appear. At the end of the process, all the machines have a library of sufficient capacity, and the output is not blocked.

(5) If buffer station $i$ is full, we will close all machines of the upstream station after processing an additional workpiece. If the system does not fail, all the machines in the downstream station will remain in operation.

(6) If there is no workpieces to be processed for buffer station $i$, the downstream station is idle and standby due to lack of material.

\section{Machine modeling}

\subsection{Multi-state machine's Markov model}

Machines have different state of operation, such as perfect performance, degraded performance and complete failure. Additionally, the transitions among non-adjacent states are also existed. The diagram of transition among non-adjacent state is shown in Fig. 1. 


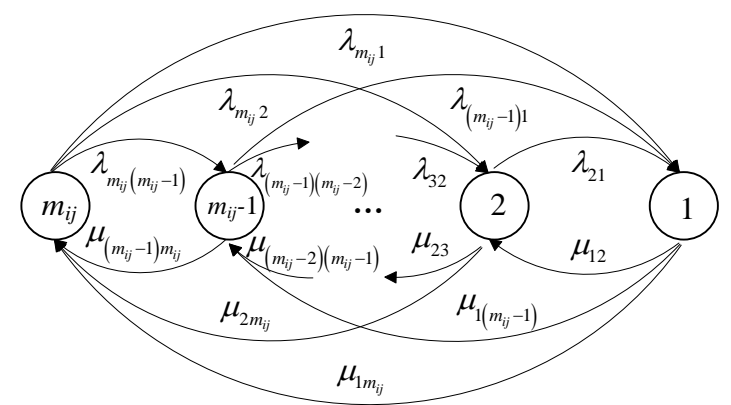

Fig. 1. Diagram of transition among non-adjacent state of machines

Because of the machine failure and repair, the state transition happens. Here, we use the state transition density $\lambda$, $\mu$ to express the failover density (failure rate) and the repair transfer density (repair rate). Then, we use Equation (1) to express a multi-state machine which has state repair and state failure between non-adjacent states.

$$
\begin{aligned}
& \frac{\mathrm{d} p_{i j m_{i}}(t)}{\mathrm{d} t}=\sum_{r=1}^{m_{j}-1} p_{i j r}(t) \mu_{r m_{j}}-p_{i j m_{i}}(t) \sum_{r=1}^{m_{j}-1} \lambda_{m_{i} r} \\
& \frac{\mathrm{d} p_{i j k}(t)}{\mathrm{d} t}=\sum_{r=k+1}^{m_{j k}} p_{i j r}(t) \lambda_{r k}-p_{i j k}(t)\left(\sum_{r=1}^{k-1} \lambda_{k r}+\sum_{r=k+1}^{m_{j}} \mu_{k r}\right)+\sum_{r=1}^{k-1} p_{i j r}(t) \mu_{r k} \\
& \frac{\mathrm{d} p_{i j 1}(t)}{\mathrm{d} t}=-p_{i j 1}(t) \sum_{r=2}^{m_{i j}} \mu_{1 r}+\sum_{r=2}^{m_{i j}} \lambda_{r 1} p_{i j r}(t)
\end{aligned}
$$

where $k=2, \ldots, m_{i j}-1, \sum_{k=1}^{m_{i j}} p_{i j k}(t)=1$ and $\quad p_{i j k}=\operatorname{Pr}\left\{G_{i j}(t)=g_{i j k}\right\}$.

Assuming that the machine is always in the optimum state $m_{i j}$, the performance value corresponding to it is $g_{i j m_{i j}}$. As the result, for Equation 1, the initial conditions are as follows:

$$
p_{i j m_{i j}}(0)=1, p_{i j\left(m_{i j}-1\right)}(0)=0, \cdots, p_{i j 2}(0)=0, \quad p_{i j 1}(0)=0
$$

Each production line only needs one steady-state solution. In Equation 1, the left side is all zero, then Equation 1 can be reduced to Equation (2).

$$
\begin{aligned}
& \sum_{r=1}^{m_{j}-1} p_{i j r}(t) \mu_{r m_{j}}-p_{i j m_{j}}(t) \sum_{r=1}^{m_{j}-1} \lambda_{m_{i j}}=0 \\
& \sum_{r=k+1}^{m_{j}} p_{i j r}(t) \lambda_{r k}-p_{i j k}(t)\left(\sum_{r=1}^{k-1} \lambda_{k r}+\sum_{r=k+1}^{m_{j}} \mu_{k r}\right)+\sum_{r=1}^{k-1} p_{i j r}(t) \mu_{r k}=0 \\
& -p_{i j 1}(t) \sum_{r=2}^{m_{j i}} \mu_{1 r}+\sum_{r=2}^{m_{j i}} \lambda_{r 1} p_{i j r}(t)=0
\end{aligned}
$$

If we use the steady-state probability of the different states of the computing machine, the calculation amount will be greatly reduced. An instance of a machine conversion with 4 different states is shown in Fig. 2. 


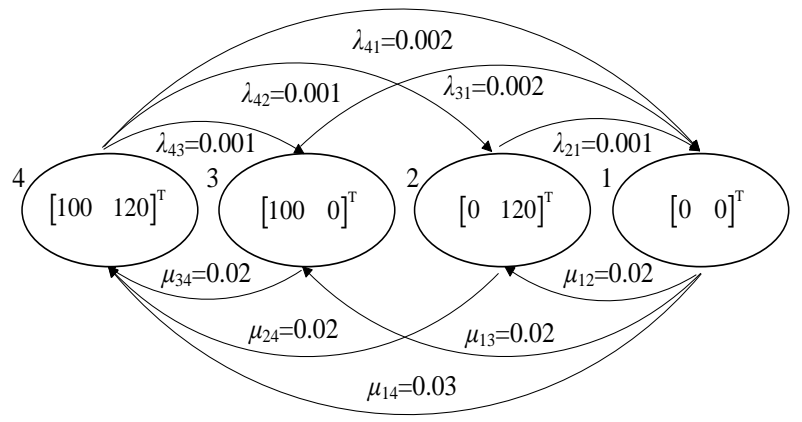

Fig. 2. Machine conversion diagram for 4 different states

In the steady state, the state conversion probability formula and theoretical steady-state probability are shown as follows:

$$
\left\{\begin{array} { l } 
{ p _ { 1 1 } \mu _ { 1 4 } + p _ { 1 2 } \mu _ { 2 4 } + p _ { 1 3 } \mu _ { 3 4 } - p _ { 1 4 } ( \lambda _ { 4 1 } + \lambda _ { 4 2 } + \lambda _ { 4 3 } ) = 0 } \\
{ p _ { 1 1 } \mu _ { 1 3 } + p _ { 1 2 } \mu _ { 2 3 } - p _ { 1 3 } ( \lambda _ { 3 1 } + \lambda _ { 3 2 } + \mu _ { 3 4 } ) + p _ { 1 4 } \lambda _ { 4 3 } = 0 } \\
{ p _ { 1 1 } \mu _ { 1 2 } - p _ { 1 2 } ( \lambda _ { 2 1 } + \mu _ { 2 3 } + \mu _ { 2 4 } ) + p _ { 1 3 } \mu _ { 3 2 } + p _ { 1 4 } \lambda _ { 4 2 } = 0 } \\
{ - p _ { 1 1 } ( \mu _ { 1 2 } + \mu _ { 1 3 } + \mu _ { 1 4 } ) + p _ { 1 2 } \lambda _ { 2 1 } + p _ { 1 3 } \lambda _ { 3 1 } + p _ { 1 4 } \lambda _ { 4 1 } = 0 }
\end{array} \Rightarrow \left\{\begin{array}{l}
p_{11}=0.0253 \\
p_{12}=0.0619 \\
p_{13}=0.0591 \\
p_{14}=0.8537
\end{array}\right.\right.
$$

Ordinary machines usually have only two states: normal operating state and full fault state. Here, we use 2 to represent the normal operating state and use 1 to represent the full fault state. State transitions happen from states 2 to 1 or from 1 to 2 . Fig. 3 shows the state transition of the normal machine.

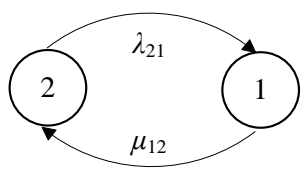

Fig. 3. State transition of ordinary machines

We reduce the state transition probability formula of general machines to Equation (3) based on Equation (1).

$$
\left\{\begin{array}{l}
\frac{\mathrm{d} p_{i j 2}(t)}{\mathrm{d} t}=p_{i j 1}(t) \mu_{12}-p_{i j 2}(t) \lambda_{21} \\
\frac{\mathrm{d} p_{i j 1}(t)}{\mathrm{d} t}=-p_{i j 1}(t) \mu_{12}+p_{i j 2}(t) \lambda_{21}
\end{array}\right.
$$

where $p_{i j 1}+p_{i j 2}=1$.

Here, we assume that the left side of Equation (3) is zero, then we can express the steady state availability of general machines as follows:

$$
p_{i j 1}=\frac{\mu_{12}}{\mu_{12}+\lambda_{21}}
$$




\subsection{State probability calculation}

We choose the adjacent three stations of the system for study to ensure the proposed method to be more general. As shown in Fig. 4, the buffer layout is given. In station $S_{i-1}, S_{i}$ and $S_{i+1}$, the machine number is $d, e$ and $f$, respectively. In station $S_{i}, M_{i 1}, M_{i e}$ represent the 1 st and $e$-th machines respectively, while $M_{i 1}^{0}$ and $M_{i e}^{0}$ denote the 1 st equivalent machine and the $e$-th equivalent machine individually. $B_{i}$ is the $i$-th buffer station.

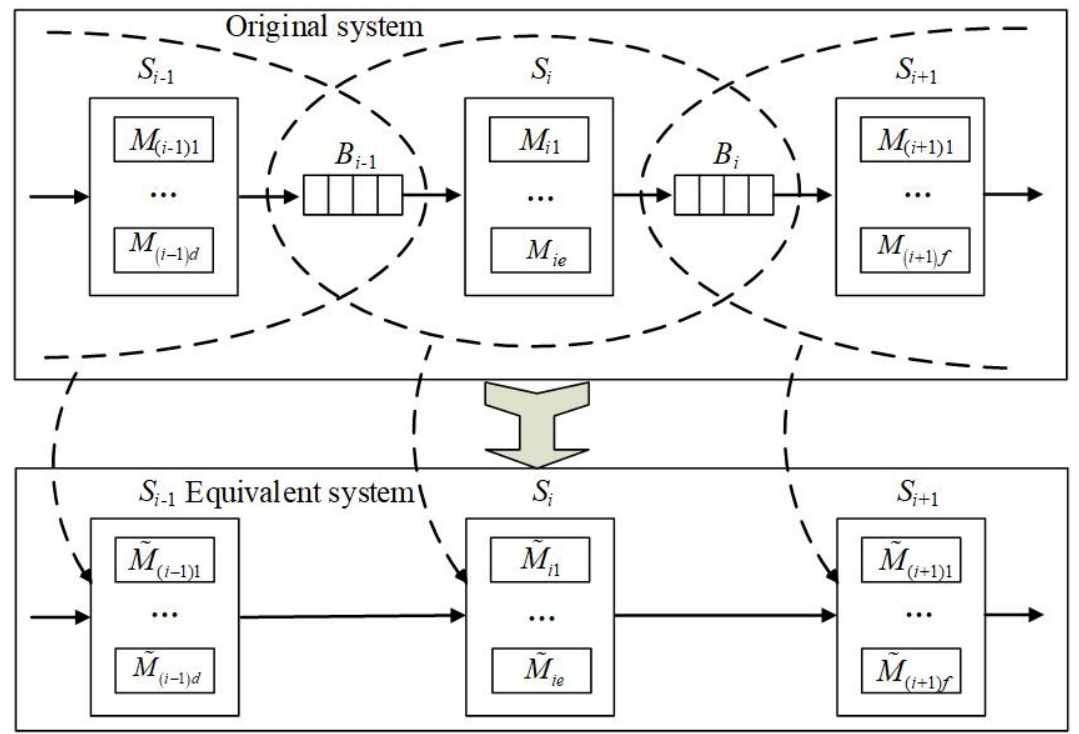

Fig. 4. A production line which has multiple buffer stations

Then, $b_{i}$ is used to represent the capacity of the $i$-th intermediate buffer $B_{i}, \omega_{i}$ is used to denote the productivity of the previous station, and $\omega_{i+1}$ is used to denote the productivity of the latter station. Next, by establishing the state transition equation of the $i$-th intermediate buffer $r$ [55], we can obtain the steady state solution. Then the probability of having $k$ $\left(k=0,1, \ldots, b_{i}\right)$ workpieces in $B_{i}$ is as follows:

$$
p_{i k}^{B}=\frac{\rho_{i}^{k}\left(1-\rho_{i}\right)}{1-\rho_{i}^{b_{i}+1}}
$$

where $\rho_{i}=\omega_{i} / \omega_{i+1}$. When $\omega_{i}=\omega_{i+1}, \quad \rho_{i}=1$ and $p_{i k}^{B}=1 /\left(b_{i}+1\right)$.

$B_{i}$ has different states during operation. These states are defines as partially empty, empty full, partially full and full, respectively. We can obtain their corresponding probabilities as follows.

State1: When $B_{i}$ is full,

$$
p_{i b_{i}}^{B}=\frac{\rho_{i}^{b_{i}}\left(1-\rho_{i}\right)}{1-\rho_{i}^{b_{i}+1}}
$$


State2: When $B_{i}$ is partially empty,

$$
p_{i b_{i}}^{B}=1-p_{i b_{i}}^{B}=\frac{1-\rho_{i}^{b_{i}}}{1-\rho_{i}^{b_{i}+1}}
$$

State3: When $B_{i}$ is empty,

$$
p_{i 0}^{B}=\frac{1-\rho_{i}}{1-\rho_{i}^{b_{i}+1}}
$$

State4: When $B_{i}$ is partially full,

$$
p_{i 0}^{B}=1-p_{i 0}^{B}=\frac{\rho_{i}\left(1-\rho_{i}^{b_{i}}\right)}{1-\rho_{i}^{b_{i}+1}}
$$

In workstation $S_{i}$, if $B_{i}$ is full, all machines will be stopped for the reason of overloading. In workstation $S_{i+1}$, if $B_{i}$ is empty, all machines will be stopped for the reason of lack of material. Thus, in workstation $S_{i}$, all machines can only work normally only if $B_{i-1}$ is partially empty or partially full. For the machines of workstation $S_{i}$, the availability of $B_{i}$ is $p_{\bar{k}}^{B}$. For the machines of workstation $S_{i}+1$, the availability of $B_{i}$ is $p_{\overline{0}}^{B}$.

Considering both the upstream and downstream buffers, machine $M_{i j}$ can have 4 conditions when it is in state $k\left(k=2, \ldots, m_{i j}\right)$ in workstation $S_{i}(2 \leq i \leq m-1)$ :

(1) The probability can be obtained when $M_{i j}$ is working normally as follows:

$$
p_{(i-1) 0}^{B} p_{i j k} p_{i b_{i}}^{B}=\frac{\rho_{i-1}\left(1-\rho_{i-1}^{b_{i-1}}\right)\left(1-\rho_{i}^{b_{i}}\right)}{\left(1-\rho_{i-1}^{b_{i-1}+1}\right)\left(1-\rho_{i}^{b_{i}+1}\right)} p_{i j k}
$$

(2) When $M_{i j}$ is in lack of material, the probability can be obtained while output is normal as follows:

$$
p_{(i-1) 0}^{B} p_{i j k} p_{i \bar{b}_{i}}^{B}=\frac{\left(1-\rho_{i-1}\right)\left(1-\rho_{i}^{b_{i}}\right)}{\left(1-\rho_{i-1}^{b_{i-1}+1}\right)\left(1-\rho_{i}^{b_{i}+1}\right)} p_{i j k}
$$

(3) When $M_{i j}$ 's input is normal, the probability can be obtained while output is blocked as follows:

$$
p_{(i-1) 0}^{B} p_{i j k} p_{i b_{i}}^{B}=\frac{\rho_{i-1}\left(1-\rho_{i-1}^{b_{i-1}}\right) \rho_{i}^{b_{i}}\left(1-\rho_{i}\right)}{\left(1-\rho_{i-1}^{b_{i-1}+1}\right)\left(1-\rho_{i}^{b_{i}+1}\right)} p_{i j k}
$$

(4) When $M_{i j}$ is in lack of material, the probability can be obtained while output is blocked as follows: 


$$
p_{(i-1) 0}^{B} p_{i j k} p_{i b_{i}}^{B}=\frac{\left(1-\rho_{i-1}\right) \rho_{i}^{b_{i}}\left(1-\rho_{i}\right)}{\left(1-\rho_{i-1}^{b_{i-1}+1}\right)\left(1-\rho_{i}^{b_{i}+1}\right)} p_{i j k}
$$

Equations (10)-(13) are used to calculate the probabilities of machine $M_{i j}$, in different conditions considering the influence of both the upstream and downstream buffer states. Equations (11)-(13) gives the probabilities that machine doesn't work normally due to machine and buffer failures. Hence, in state $k$, the steady-state probability of machine $M_{i j}$ is as follows:

$$
\beta_{i j k}=\frac{\rho_{i-1}\left(1-\rho_{i-1}^{b_{i-1}}\right)\left(1-\rho_{i}^{b_{i}}\right)}{\left(1-\rho_{i-1}^{b_{i-1}+1}\right)\left(1-\rho_{i}^{b_{i}+1}\right)} p_{i j k}
$$

In state $k$, the probability of machine $M_{i j}$ not being able to be maintained because of being blocked, lack of material or machine failures can be obtained as follows:

$$
\bar{p}_{i j k}=\left(1-\frac{\rho_{i-1}\left(1-\rho_{i-1}^{b_{i-1}}\right)\left(1-\rho_{i}^{b_{i}}\right)}{\left(1-\rho_{i-1}^{b_{i-1}+1}\right)\left(1-\rho_{i}^{b_{i}+1}\right)}\right) p_{i j k}
$$

It's assumed that all the machines have enough workpieces in the first workstation. Then, we can obtain the actual steady-state probability $\beta_{1 j k}$ of machine $M_{1 j}$ in state $k\left(2 \leq k \leq m_{i j}\right)$ as shown in Equation (16). Further, we can also obtain the non-steady-state probability $\bar{p}_{1 j k}$ of machine $M_{1 j}$ in state $k\left(2 \leq k \leq m_{i j}\right)$ as shown in Equation (16).

$$
\begin{aligned}
& \beta_{1 j k}=\frac{1-\rho_{1}^{b_{1}}}{1-\rho_{1}^{b_{1}+1}} p_{1 j k} \\
& \bar{p}_{1 j k}=\frac{\rho_{1}^{b_{1}}\left(1-\rho_{1}\right)}{1-\rho_{1}^{b_{1}+1}} p_{1 j k}
\end{aligned}
$$

In the last workstation, we assume that all the machines have enough stock space to ensure smooth output. Then, in state $k\left(2 \leq k \leq m_{i j}\right)$, we can obtain the actual steady-state probability and non-steady-state probability of machine $M_{m j}$ of workstation $S_{m}$ as follows:

$$
\begin{aligned}
& \beta_{m j k}=\frac{\rho_{m-1}\left(1-\rho_{m-1}^{b_{m-1}}\right)}{1-\rho_{m-1}^{b_{m-1}+1}} p_{m j k} \\
& \bar{p}_{m j k}=\frac{1-\rho_{m-1}}{1-\rho_{m-1}^{b_{m-1}+1}} p_{m j k}
\end{aligned}
$$

The probability of failure of the machine $M_{i j}$ without the consideration of the influence of buffer states is as follows:

$$
p_{i j 1}=1-\sum_{k=2}^{m_{i j}} p_{i j k}
$$

For the machine $M_{i j}$, the failure probability equivalence considering the buffer state is as follows:

$$
\beta_{i j 1}=1-\sum_{k=2}^{m_{i j}} \beta_{i j k}=p_{i j 1}+\sum_{k=2}^{m_{i j}} \bar{p}_{i j k}
$$


Therefore, it can be conclude that $M_{i j}$ is equivalent to $M_{i j}$ by equations (14)-(19). Then we can convert the original production system into an equivalent system which is shown in Fig. 4.

\subsection{Machine UGF calculation}

When there are enough machines, buffer stations, auxiliary equipment and logistics equipment in the whole system, the state space will be increasing exponentially. This problem is very difficult to solve. Here, we can use UGF method as an effective tool. Considering the effects of buffer states, the function $u_{i j}(z)$, which represents the performance rate of machine $M_{i j}$, can be derived from the following equation:

$$
u_{i j}(z)=\sum_{k=1}^{m_{i j}} \beta_{i j k} z^{g_{i j k}}
$$

where $\beta_{i j k}$ is the actual steady-state probability and $\boldsymbol{g}_{i j k}$ is the performance vector.

When the machine is processing different workpieces, it also has different steady-state probabilities, which can be represented by vectors, so the early $U$ function evolves to:

$$
u_{i j}(z)=\sum_{k=1}^{m_{i j}} \beta_{i j k} z^{g_{i j k}}
$$

where $\boldsymbol{g}_{i j k}$ is the performance vector and $\boldsymbol{\beta}_{i j k}$ is the actual steady-state probability vector corresponding to the performance vector $\boldsymbol{g}_{i j k}$

\section{System modeling}

\subsection{System UGF calculation}

The probability and performance rate for each state are represented by the same vector size as the artifact type. Therefore, we can divide the equivalent production system into independent serial subsystems and parallel subsystems.

Then we can obtain the U-function $\mathcal{U}_{s}^{\%}(z)$ of the system by recursive decomposition and assess the final result by operators (parallel and serial) and equivalent modules.

$$
\begin{aligned}
& U_{s}^{\%}(z)=\otimes\left(U_{1}^{\%}(z), U_{2}^{\%}(z)\right)
\end{aligned}
$$

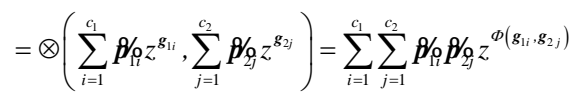

where (1) $\mho_{1}(z), \mho_{2}(z)$ are the $u$-functions of equivalent subsystem 1, 2; (2) $c_{1}, c_{2}$ are the state number in equivalent subsystem 1,2 ; (3) $\beta_{11}^{0}, \beta_{2 j}^{0}$ are the probability vectors which are corresponding to performance vector $\boldsymbol{g}_{1 i}, \boldsymbol{g}_{2 j} . \quad \beta_{11}^{\prime}=\left[\begin{array}{llll}\beta_{11}^{\gamma} & \beta_{11}^{\gamma} & \mathrm{L} & \beta_{11}^{\gamma}\end{array}\right]^{\mathrm{T}}$, $\beta_{2 j}^{\prime}=\left[\begin{array}{llll}\beta_{2 j}^{\circ} & \beta_{2 j}^{\prime} & L & \beta\end{array}\right]_{2 j}^{\mathrm{T}}$; (4) $\mathcal{U}_{s}^{\%}(z)$ is the $u$-functions of the entire system $S$ composed of equivalent subsystem 1 and 2 .

Serial composition operator in vector is defined as: 


$$
\begin{aligned}
& \sigma\left(\mathcal{U}_{1}^{\%}(z), \mathcal{U}_{2}^{\%}(z)\right) \\
& =\sigma\left(\sum_{i=1}^{c_{1}} \boldsymbol{\beta}_{11} z^{\boldsymbol{g}_{1 i}}, \sum_{j=1}^{c_{2}} \boldsymbol{\beta}_{2 j}^{0} z^{\boldsymbol{g}_{2 j}}\right)=\sum_{i=1}^{c_{1}} \sum_{j=1}^{c_{2}} \boldsymbol{\beta}_{1 i}^{0} \boldsymbol{\beta}_{2 j}^{\circ} z^{\min \left(\boldsymbol{g}_{1,}, \boldsymbol{g}_{2 j}\right)}
\end{aligned}
$$

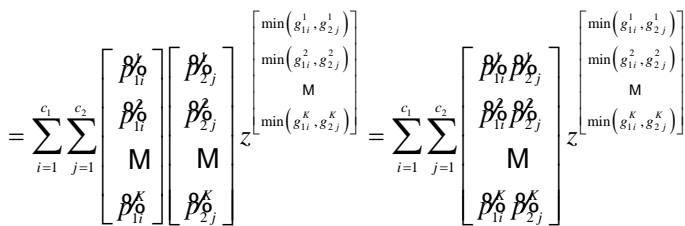

Parallel composition operator in vector is defined as:

$$
\begin{aligned}
& \pi\left(U_{1}^{\mathscr{O}}(z), U_{2}^{\mathscr{\%}}(z)\right) \\
& =\pi\left(\sum_{i=1}^{c_{1}} \boldsymbol{\beta}_{1 i}^{0} z^{\boldsymbol{g}_{1 i}}, \sum_{j=1}^{c_{2}} \boldsymbol{\beta}_{2 j}^{\prime} \mathbf{g}^{\boldsymbol{g}_{2 j}}\right)=\sum_{i=1}^{c_{1}} \sum_{j=1}^{c_{2}} \boldsymbol{\beta}_{1 i}^{0} \boldsymbol{\beta}_{2 j}^{\prime} \mathrm{z}^{\boldsymbol{g}_{1 i}+\boldsymbol{g}_{2 j}}
\end{aligned}
$$

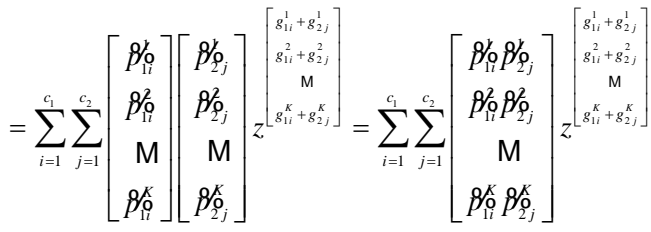

For a serial-parallel production system, the process of calculating the U-function of the system is as follows:

(1) Considering the buffer state, the structure of the equivalent system is decomposed.

(2) The UGF of all parallel equivalent subsystems is calculated by parallel composition operator and equation (21).

(3) The serial-parallel hybrid production system can be simplified to the serial-parallel hybrid production system.

(4) By a series of combinatorial operators, the $\mho_{s}^{\%}(z)$ of the entire equivalent system is calculated which is shown in Fig. 5.

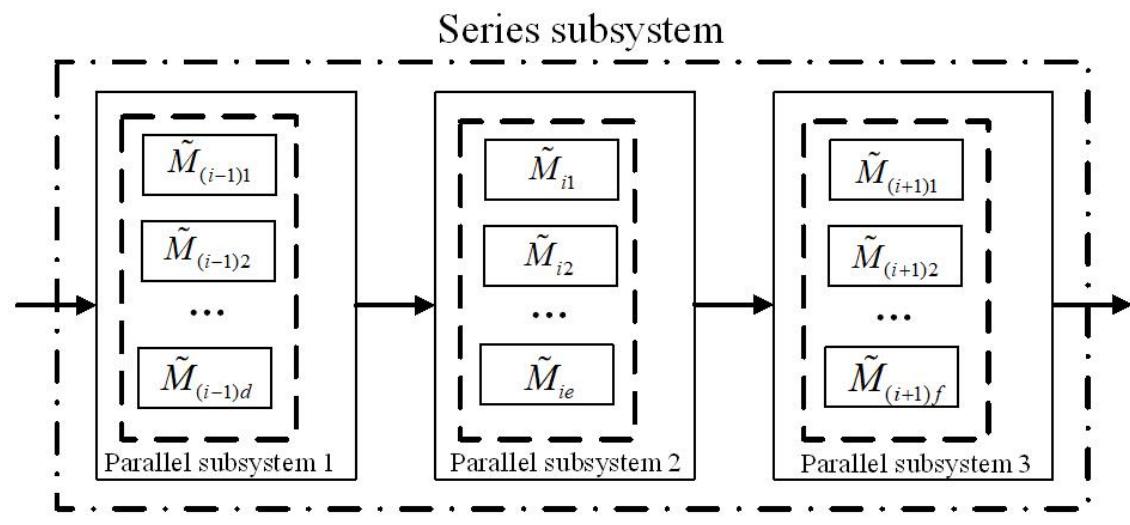

Fig. 5. Decomposition of systems.

\subsection{System multi-state reliability}

For a multi-state production system, we define a parameter by improving the classical reliability index to depict its reliability behavior. Reliability, which is a performance 
indicator that the system meets certain performance levels, can be evaluated by the operator $\delta_{\mathrm{E}}$ using the system theoretical productivity $\boldsymbol{E}_{S}(\mathrm{z})$.

$$
\boldsymbol{E}_{s}(z)=\delta_{\mathrm{E}}\left(\bigcup_{s}^{\mathcal{O}}(z)\right)=\delta_{\mathrm{E}}\left(\sum_{i=1}^{M} \boldsymbol{p}_{i}^{s} z^{\boldsymbol{g}_{i}^{s}}\right)=\sum_{i=1}^{M} \boldsymbol{p}_{i}^{s} \boldsymbol{g}_{i}^{s}
$$

\subsection{System structural complexity}

State identification and calculation is the fundamental for studying the structural complexity of a production system, which depends on the research purpose and granularity of the production system. Different focuses will result in different state recognitions and state number. Actually, we generally pay more attention to the overall performance of the production system. From the standpoint of operability, events related to the production capacity of the production system are taken as states utilized to complexity calculation. For example, full-operation state, failure state, and a variety of discrete degraded states between them.

Let the system have a total of $M$ states, and the state vector space is

$$
\boldsymbol{g}_{s}=\left\{\boldsymbol{g}_{1}^{s}, \boldsymbol{g}_{2}^{s}, \mathbf{L}, \boldsymbol{g}_{M}^{s}\right\}
$$

where the corresponding probability vector space is

$$
\boldsymbol{p}_{s}=\left\{\boldsymbol{p}_{1}^{s}, \boldsymbol{p}_{2}^{s}, \mathrm{~L}, \boldsymbol{p}_{M}^{s}\right\}
$$

the $i$-th state vector and probability vector respectively are

$$
\boldsymbol{g}_{i}^{s}=\left[\begin{array}{llll}
g_{i 1}^{s} & g_{i 2}^{s} & \mathrm{~L} & g_{i K}^{s}
\end{array}\right]^{\mathrm{T}}, \quad \boldsymbol{p}_{i}^{\mathrm{s}}=\left[\begin{array}{llll}
p_{i 1}^{s} & p_{i 2}^{s} & \mathrm{~L} & p_{i K}^{s}
\end{array}\right]^{\mathrm{T}}
$$

Entropy is a measure of information content and its eigenvalues represent the degree of uncertainties of the existing and operating states of the system. It is widely used to measure uncertainties in deterministic phenomenon. The greater the information entropy is, the greater the uncertainties of the system is. For a particular system, its information entropy is a certain value. According to the definition of information entropy, the state entropy of the production system can be defined as

$$
H_{s}=-\sum_{i=1}^{M} \sum_{j=1}^{K} p_{i j}^{s} \log _{2} p_{i j}^{s}
$$

where $p_{i j}^{s}$ is the probability corresponding to the state $g_{i j}^{s}, 1 \leq i \leq M$.

It can be seen from the model that the control of the resource states from the micro level is transformed into the control of the output states of the entire production system on the macro level. This not only makes it possible to establish a more accurate measurement model for the production system complexity, but also to grasp the complexity of the entire system from a higher level. That is, if the entire production system is viewed as a whole, it is only necessary to plan and control the states of the entire system without having to identify the states of individual production resources.

In addition, according to the information entropy theory, the larger the system entropy value, the greater the uncertainties and unpredictability of the system states, and the more unstable the system performs. At the same time, the amount of information needed to understand the system is greater. Using Equation (14), we can accurately measure the complexity and quantitatively analyze the states of the production systems, that is, we can 
more precisely understand the structural complexity of the production system to evaluate the system design.

\section{Buffer allocation}

\subsection{Optimal allocation model}

Generally, for the buffer capacity configuration problem, we can establish different optimization models according to different optimization goals, such as solve the minimum buffer capacity under the premise of satisfying the average productivity of the system, or given the total buffer capacity, optimize the maximum system productivity. When establishing the buffer optimization model, what kind of indicator is selected as the objective function can be reasonably determined according to practical requirements. In fact, in the globalized and interrelated market, demand fluctuation which is along with the requirements of low cost, high product quality, high customization and short lead time, may lead to a rise in production complexity. For a production system, its structural complexity is related to the configuration and structure of itself, the type and number of its machining parts, the type of elements of itself (such as material handling vehicles, buffer stations, machines) and their interdependencies and interconnections.

In order to ultimately design and plan a more productive and predictive manufacturing system, the nonlinear behavior of the production system should be understood and controlled $[29,30]$. So, a mathematical model for buffer capacity optimization allocation based on the reliability and complexity of the production system is established in this paper.

$$
\begin{gathered}
\min f\left(b_{1}, \mathrm{~L}, b_{m-1}\right)=-\alpha \sum_{j=1}^{K} E_{j}^{s}+\beta H_{s} \\
\text { s.t. } \sum_{i=1}^{m-1} b_{i} \leq B \\
b_{i} \geq b_{\mathrm{MIN}} \\
E_{j}^{s} \geq E_{\mathrm{MIN}} \quad j=1,2, \mathrm{~L}, m-1 \\
b_{i}, B, b_{\mathrm{MIN}} \in Z^{+} \quad E_{j}^{s}, E_{\mathrm{MIN}} \in R^{+}
\end{gathered}
$$

where $E_{j}^{s}$ is the $j$-th component of system theoretical production rate, that is, the theoretical productivity that can be achieved when the system is machining part $P_{j} . \sum E_{j}^{s}$ represents the sum of theoretical production rates that can be achieved when machining part family $\boldsymbol{P}=\left\{P_{1}, P_{2}, \cdots, P_{K}\right\} ; \alpha$ and $\beta$ are weight coefficients. $b_{i}$ represents the capacity of the $i$-th buffer station. $B$ denotes the maximum total buffer capacity allowed in the system. $b_{\text {MIN }}$ denotes the minimum buffer capacity allocated to each buffer station and $b_{\text {MIN }}=4$ in discrete systems [56]. $E_{\mathrm{MIN}}$ is the minimum theoretical productivity that should be achieved when machining each part. Constraint (28) is the upper bound of total buffer capacity. Constraint (29) is the lower bound of each buffer capacity. Constraint (30) denotes that the machining capability for each part is not less than the minimum theoretical productivity allowed. 


\subsection{Optimal allocation algorithm}

Buffer capacity allocation is a typical combinatorial optimization problem. It can be seen from the mathematical model established in the previous section that the model is an integer linear programming model. When the number of machining equipment and buffers to be allocated increase, it is difficult to solve the problem and obtain an optimal solution in polynomial time. In this paper, we utilize the genetic algorithm to find the optimal solution of buffer allocation model. The related settings and processes are as follows:

(1) Chromosome representation: in this paper, we utilize integer to represent the parameter space. Suppose that there are nine buffer stations in a production line that need to be allocated capacity, and the order in which the parts flow through the buffer is $b_{1} \rightarrow b_{9}$, then the encoded buffer stations are represented as the chromosome shown in Fig. $\mathbf{6}$.

\begin{tabular}{|l|l|l|l|l|l|l|l|l|}
\hline$b_{1}$ & $b_{2}$ & $b_{3}$ & $b_{4}$ & $b_{5}$ & $b_{6}$ & $b_{7}$ & $b_{8}$ & $b_{9}$ \\
\hline
\end{tabular}

Fig. 6. Schematic diagram of chromosome structure.

(2) Population initialization: define the population size $N_{\text {pop_size }}$ and randomly generate $N_{\text {pop_size }}$ individuals. Since the number of parts that can be stored in the buffer stations can only be a positive integer, each gene in the chromosome is a positive integer satisfying $b_{\mathrm{MIN}} \leq b_{i} \leq B$, wherein the value of the first gene position is a natural number that is not greater than $B-(m-1)$, the others are not greater than $B-(m-1-i)-\sum b_{i}$.

(3) Fitness function computation: firstly, calculate the theoretical production rate $E_{j}^{s}$ when machining $P_{j}$, sum all the theoretical production rates of part family $\boldsymbol{P}=\left\{P_{1}, P_{2}, \cdots, P_{K}\right\}$, get the system state entropy $H_{s}$ based on section 2 - 4, and then define the fitness function as follows:

$$
\begin{aligned}
F\left(b_{1}, \mathrm{~L}, b_{m-1}\right)= & f\left(b_{1}, \mathrm{~L}, b_{m-1}\right)+ \\
& \xi\left(\max \left(0, \sum_{i=1}^{m-1} b_{i}-B\right)+\max \left(0, b_{\text {MIN }}-b_{i}\right)+\max \left(0, E_{\text {MIN }}-E_{j}^{s}\right)\right)
\end{aligned}
$$

where $\xi$ is a penalty factor. std denotes the standard deviation function. $P_{\mathrm{INF}}$ is the number of infeasible solutions, and accordingly the number of feasible solutions is $P_{\mathrm{F}}=N_{\text {pop_size }}-P_{\mathrm{INF}}$. $\Gamma$ is the feasible collection. $\xi$ can be obtained by [56]

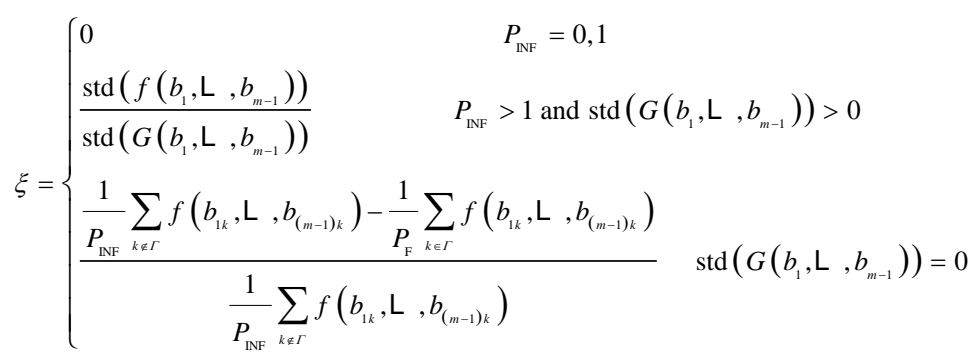


where std is the standard deviation function, $G\left(b_{1}, \mathrm{~L}, b_{m-1}\right)$ refers to the degree that the solution violates the boundary conditions, and define it as follows:

$$
G\left(b_{1}, \mathrm{~L}, b_{m-1}\right)=\left(\sum_{i=1}^{m-1} b_{i}-B\right)+\left(b_{\mathrm{MIN}}-b_{i}\right)+\left(E_{\mathrm{MIN}}-E_{j}^{s}\right)
$$

(4) Genetic operators: the selection operation uses a combination of elite preservation strategy and roulette selection. Individuals with the highest fitness use elite conservation strategy that replicate directly to the next generation without crossover and mutation. Using the roulette method, the probability of selecting each individual is calculated according to the fitness of the individual and the sum of the group fitness. Consequently, a new population is generated.

Use the adaptive strategy to control the crossover probability and mutation probability:

$$
p= \begin{cases}p+\frac{\Delta p\left(N_{\mathrm{MAX}}-i\right)}{N_{\mathrm{MAX}}} & \bar{f}_{i}>\bar{f}_{i-1} \\ p-\frac{\Delta p\left(N_{\mathrm{MAX}}-i\right)}{N_{\mathrm{MAX}}} & \bar{f}_{i}<\bar{f}_{i-1} \\ p+\frac{2 \Delta p\left(N_{\mathrm{MAX}}-i\right)}{N_{\mathrm{MAX}}} & \bar{f}_{i}=\bar{f}_{i-1}\end{cases}
$$

where $\bar{f}_{i}, \bar{f}_{i-1}$ are the average fitness of the $i$-th and (i-1)-th generation population; $0.5 \leq p_{c} \leq 1.0, \quad 0 \leq p_{m} \leq 0.2, \Delta p_{c}$ and $\Delta p_{m}$ need to be determined according to the actual situation.

(5) Producing a new generation: after a series of genetic operations such as selection, crossover and mutation, a new generation is produced and its size is still $N_{\text {pop_size }}$.

(6) Termination: the genetic algorithm is an iterative process. Each iteration must performs fitness calculation, selection, intersection, and mutation. The algorithm terminates when either of the following two conditions are met: (1) the number of iterations reaches the set value; (2) the fitness of the best individuals remains constant or within the specified minimum deviation for $N_{\text {CoN }}$ successive generations.

\section{Case study}

In this section, we review a production line in South China that is used to machine two different engine heads. The case is shown in Fig. 7.

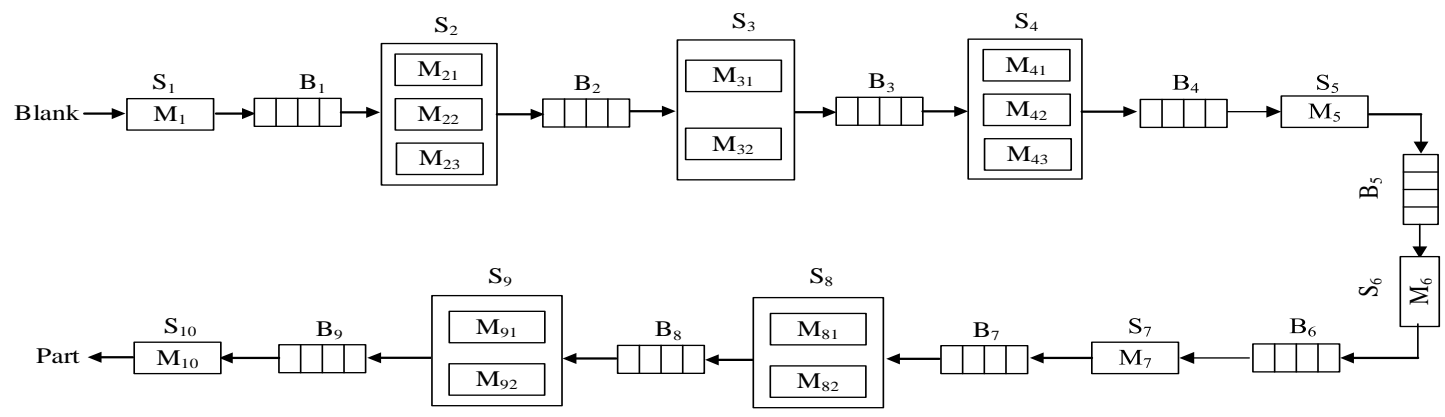

Fig. 7. Engine head production line with 10 workstations and 9 intermediate buffers. 
Here, we assume that the sojourn times follow exponential distribution for all the machines and auxiliary equipment. By Equations 2, we can obtain the steady-state availability of each machine in accordance with the state transition diagram, repair rates and failure rates. In Table 1, we give the parameters of each machine.

In the production line, there are 10 workstations $\left(S_{1}-S_{10}\right)$ and 9 intermediate buffer stations $\left(B_{1}-B_{9}\right)$. The detail information is as follows.

(1) There is only one machine $M_{1}$ in workstation $S_{1}$.

(2) There are two identical machines in workstations $S_{3}, S_{8}$ and $S_{9}$. The two identical machines are parallel, that are $\left(M_{31}, M_{32}\right),\left(M_{81}, M_{82}\right),\left(M_{91}, M_{92}\right)$.

(3) There are three identical machines in workstations $S_{2}$ and $S_{4}$. That are $\left(M_{21}, M_{22}, M_{23}\right)$ and $\left(M_{41}, M_{42}, M_{43}\right)$.

(4) Else, $S_{5}, S_{6}, S_{7}$ and $S_{10}$ belong to auxiliary workstations.

(5) The cleaning machines are $M_{5}$ and $M_{10}$.

(6) The press-fit machine is $M_{6}$.

(7) The leak detector is $M_{7}$.

The capacity of nine buffer stations is represented by $b_{1}-b_{9}$.

It is assumed that the stay time of all machines and auxiliary equipment is subject to an exponential distribution.

According to the failure rate, repair rate and state transition diagram, we utilize Equation 2 to obtain the steady state availability of each machine. In Table 1, the parameters of each machine are shown.

Table 1. Parameters of each machine

\begin{tabular}{|c|c|c|c|c|c|c|c|c|c|}
\hline \multirow{3}{*}{ Workstation } & \multirow{2}{*}{ Machine } & \multicolumn{2}{|c|}{$\begin{array}{c}\text { Productivity } \\
\omega /\left(\mathrm{pcs} \cdot \mathrm{h}^{-1}\right)\end{array}$} & \multirow{2}{*}{$p_{i j}$} & \multirow{2}{*}{ Workstation } & \multirow{2}{*}{ Machine } & \multicolumn{2}{|c|}{$\begin{array}{c}\text { Productivity } \\
\omega /\left(\mathrm{pcs} \cdot \mathrm{h}^{-1}\right)\end{array}$} & \multirow{2}{*}{$p_{i j}$} \\
\cline { 3 - 4 } & & $P_{1}$ & $P_{2}$ & & & & $P_{1}$ & $P_{2}$ & \\
\hline \hline$S_{1}$ & $M_{1}$ & 18 & 20 & 0.9405 & $S_{6}$ & $M_{6}$ & 50 & 50 & 0.8404 \\
\hline$S_{2}$ & $M_{2}$ & 7 & 8 & 0.9228 & $S_{7}$ & $M_{7}$ & 60 & 60 & 0.8429 \\
\hline$S_{3}$ & $M_{3}$ & 10 & 11 & 0.9376 & $S_{8}$ & $M_{8}$ & 11 & 13 & 0.9481 \\
\hline$S_{4}$ & $M_{4}$ & 7 & 7 & 0.9152 & $S_{9}$ & $M_{9}$ & 14 & 15 & 0.9477 \\
\hline$S_{5}$ & $M_{5}$ & 35 & 35 & 0.8615 & $S_{10}$ & $M_{10}$ & 35 & 35 & 0.8615 \\
\hline
\end{tabular}

In this section, we use the genetic algorithm-based buffer capacity allocation optimization algorithm proposed in the previous section to optimize the nine buffer capacity in the cylinder head production line. We set $\alpha=1, \beta=5$ in this section. The total buffer size to be allocated is $B=300$, and the theoretical production rate of each part is not less than $6 \mathrm{pcs} / \mathrm{h}$. The problem may be stated mathematically as follows:

$$
\begin{gathered}
\min f\left(b_{1}, \mathrm{~L}, b_{9}\right)=-\left(E_{1}^{s}+E_{2}^{s}\right)+5 H_{s} \\
\text { s.t. } \sum_{i=1}^{m-1} b_{i} \leq 200 \quad b_{i} \geq 4 \quad b_{i} \in Z^{+} \\
E_{1}^{s} \geq 6 \quad E_{2}^{s} \geq 6
\end{gathered}
$$

Parameter definitions: crossover probability $p_{c}=0.5$, mutation probabilities are $p_{m}=0.2$, $\Delta p_{c}=0.02$ and $\Delta p_{m}=0.002$ respectively, number of iterations are $N_{\mathrm{MAX}}=200$ and $N_{\mathrm{CON}}=50$. Considering the random nature of the genetic algorithm, we select the population size 
$N_{\text {pop_size }}=20,50$, and 100 to run independently respectively. The optimal results in each case are shown in Table 2.

Table 2. Optimal allocation of buffer capacity

\begin{tabular}{|c|c|c|c|c|c|c|c|c|c|c|}
\hline \multirow{2}{*}{$N_{\text {pop_size }}$} & \multicolumn{7}{|c|}{ Buffer capacity } & \multirow{2}{*}{ Objective value } \\
\cline { 2 - 11 } & $B_{1}$ & $B_{2}$ & $B_{3}$ & $B_{4}$ & $B_{5}$ & $B_{6}$ & $B_{7}$ & $B_{8}$ & $B_{9}$ & \\
\hline \hline 20 & 34 & 37 & 40 & 14 & 38 & 39 & 41 & 23 & 34 & 7.2262 \\
\hline 50 & 34 & 37 & 44 & 16 & 40 & 39 & 39 & 23 & 28 & 7.2045 \\
\hline $\mathbf{1 0 0}$ & $\mathbf{3 4}$ & $\mathbf{3 7}$ & $\mathbf{4 5}$ & $\mathbf{1 5}$ & $\mathbf{3 9}$ & $\mathbf{3 9}$ & $\mathbf{4 0}$ & $\mathbf{2 3}$ & $\mathbf{2 8}$ & $\mathbf{7 . 2 0 0 6}$ \\
\hline
\end{tabular}

Table 1 shows that when we set $B_{1}(34), B_{2}(37), B_{3}(45), B_{4}(15), B_{5}(39), B_{6}(39), B_{7}(40)$, $B_{8}(23), B_{9}(28)$, system performance is optimal.

\section{Conclusions}

\subsection{Summary}

The purpose of buffer allocation is to solve the problem of allocating a reasonable size of intermediate buffer for production system. Buffer allocation problem is very significant to production system design in terms of limiting the spread of logistics interruption and improving the average throughput of the system.

This paper constructs a buffer state function, analyzes the impact of buffer capacity on the steady-state availability of production equipment, and uses the extended vector UGF to calculate the $u$ function of the whole system. Two indicators including multi-state reliability, structural complexity and their corresponding measures are developed respectively. Consequently, we establish the mathematical model of buffer capacity distribution and develop a GA-based optimization algorithm.

This method can not only effectively overcome the "state dimension explosion" and equipment state coupling problem, but also greatly reduces the solving complexity while realizing the accurate description of the system state. It is very suitable for analysis of the reliability and complexity of large-scale multi-state complex production systems, as well as the system performance and structure synthesis and optimization.

\subsection{Future work}

There are many indicators for assessing the performance of production systems. In general, we usually choose different indicators according to our focus and purpose. For example, the indicators which we use to evaluate the multi-state reliability of a repairable production system include, in addition to the system theoretical production rate mentioned in this paper, system steady-state availability, system efficient production rate, system production loss, system utilization rate, etc. Besides structural complexity, there are also system decision-making complexity and operational complexity apt for complexity assessment. The proposed optimization model, combining system theoretical production rate and system structural complexity, is a multiple objective optimization problem. But we transform the multiple objective optimization problem into a single objective model by 
simple weighting calculations. Although this can reduce the complexity of the solution, it will also have a certain randomness due to the unreasonable weight selection. Therefore, how to reasonably select evaluation indicators and develop a more objective and reasonable multi-objective intelligent optimization algorithm is a key issue to further improve the rationality of buffer allocation.

\section{References}

[1] Gershwin S.B., "The future of manufacturing systems engineering," International Journal of Production Research, vol. 56, no. 1-2, pp. 224-237, Nov. 2017.Article (CrossRef Link)

[2] Shi, C., and S.B. Gershwin, "A segmentation approach for solving buffer allocation problems in large production systems," International Journal of Production Research, vol. 54, no. 20, pp. 6121-6141, May 2014. Article (CrossRef Link)

[3] Enginarlar E., J.S. Li, S.M. Meerkov and R.Q. Zhang, "Buffer capacity for accommodating machine downtime in serial production lines," in Proc. of the 40th IEEE Conference on Decision and Control (Cat. No.01CH37228), vol. 2, pp. 1947-1952, 2001. Article (CrossRef Link)

[4] Sabuncuoglu I., E. Erel and Y. Gocgun, "Analysis of serial production lines: characterisation study and a new heuristic procedure for optimal buffer allocation," International Journal of Production Research, vol. 44, no. 13, pp. 2499-2523, Jul 2006. Article (CrossRef Link)

[5] Konishi K, "A tuning strategy to avoid blocking and starving in a buffered production line," European Journal of Operational Research, vol. 200, no. 2, pp. 616-620, Jan 2010.

Article (CrossRef Link)

[6] Staley D.R. and D.S. Kim, "Experimental results for the allocation of buffers in closed serial production lines," International Journal of Production Economics, vol. 137, no. 2, pp. 284-291, Jun 2012. Article (CrossRef Link)

[7] Sana S.S., "Preventive maintenance and optimal buffer inventory for products sold with warranty in an imperfect production system," International Journal of Production Research, vol. 50, no. 23, pp. 6764-6774, Jan 2011. Article (CrossRef Link)

[8] Kose S.Y. and O. Kilincci, "Hybrid approach for buffer allocation in open serial production lines,” Computers \& Operations Research, vol. 60, pp. 67-78, Aug 2015. Article (CrossRef Link)

[9] Gan S.Y., Z.S. Zhang, Y.F. Zhou and J.F. Shi, "Joint optimization of maintenance, buffer, and spare parts for a production system," Applied Mathematical Modelling, vol. 39, no. 19, pp. 6032-6042, Jan 2015. Article (CrossRef Link)

[10] Sabry S., M. Tom and D. Viatcheslav, "Asymmetrical buffer allocation in unpaced merging assembly lines,” Computers \& Industrial Engineering, vol. 109, pp. 211-220, May 2017. Article (CrossRef Link)

[11] Gan S.Y., Z.H. Zhang, Y.F. Zhou and J.F. Shi, "Intermediate buffer analysis for a production system,” Applied Mathematical Modelling, vol.37, pp. 8785-8795, Nov 2013. Article (CrossRef Link)

[12] Ng A.H.C., S. Sabry and J. Bernedixen, "Studying unbalanced workload and buffer allocation of production systems using multi-objective optimisation," International Journal of Production Research, vol. 55, no. 24, pp. 7435-7451, Aug 2017. Article (CrossRef Link)

[13] Zhang J.Y., L.Y. Xie and B. Li, "Reliability Analysis of Non-series Manufacturing System Based on Petri Nets,” Journal of Mechanical Engineering, vol. 45, no. 12, pp. 95-101, Jan 2009. Article (CrossRef Link)

[14] Lin Y.K., C.F. Huang and P.C. Chang, "System reliability evaluation of a touch panel manufacturing system with defect rate and reworking," Reliability Engineering and System Safety, vol, 118, pp. 51-60, Oct 2013. Article (CrossRef Link)

[15] Fitouhi M.C., M. Nourelfath and S.B. Gershwin, "Performance evaluation of a two-machine line with a finite buffer and condition-based maintenance," Reliability Engineering and System Safety, vol. 166, pp. 61-72, Apr 2017. Article (CrossRef Link) 
[16] Sakurahara T., Schumock G., Reihani S., Kee E. and Mohaghegh Z., "Simulation-Informed Probabilistic Methodology for Common Cause Failure Analysis,” Reliability Engineering and System Safety, vol. 185, pp. 84-99, Dec 2018. Article (CrossRef Link)

[17] Qiu Z, R. Huang, X. Wang and W. Qi, "Structural reliability analysis and reliability-based design optimization: recent advances,” Science China Physics, Mechanics \& Astronomy, vol. 56, no. 9, pp.1611-1618, Sep 2013. Article (CrossRef Link)

[18] Bukowski L., "System of systems dependability-Theoretical models and applications examples," Reliability Engineering and System Safety, vol. 151, pp. 76-92, Nov 2015. Article (CrossRef Link)

[19] Li L., Hang J., Gao Y. and Mu C, “Using an Integrated Group Decision Method Based on SVM, TFN-RS-AHP, and TOPSIS-CD for Cloud Service Supplier Selection,” Mathematical Problems in Engineering, vol. 2017, pp. 1-15, Jan 2017. Article (CrossRef Link)

[20] Peng W.W., L.J. Shen, Y. Shen and Q.Z. Sun, "Reliability analysis of repairable systems with recurrent misuse-induced failures and normal-operation failures," Reliability Engineering and System Safety, vol. 171, pp. 87-98, Nov 2017. Article (CrossRef Link)

[21] Youssef A.M.A., A. Mohi, and H. A. EIMaraghy, "Availability assessment of multi-state manufacturing systems using universal generating function,” CIRP Annals- Manufacturing Technology, vol. 55, no. 1, pp. 445-448, Mar 2006. Article (CrossRef Link)

[22] Song X.G., Z.J. Zhai and Y.D. Liu, "A stochastic approach for the reliability evaluation of multi-state systems with dependent components," Reliability Engineering and System Safety, vol. 170, pp. 257-266, Nov 2017.Article (CrossRef Link)

[23] Mo Y.C., Y. Liu and L.R. Cui, "Performability analysis of multi-state series-parallel systems with heterogeneous components,” Reliability Engineering and System Safety, vol. 171, pp. 48-56, 2018. Article (CrossRef Link)

[24] Youssef A.M.A. and H.A. EIMaraghy, "Performance analysis of manufacturing systems composed of modular machines using the universal generating function," Journal of manufacturing systems, vol. 27, pp.55-69, May 2008. Article (CrossRef Link)

[25] Li L.H., Hang J.C., Sun H.X. and Wang L., “A conjunctive multiple-criteria decision-making approach for cloud service supplier selection of manufacturing enterprise,” Advances in Mechanical Engineering, vol. 9, no. 3, pp. 1-15, Mar 2017. Article (CrossRef Link)

[26] Azadeh A., S.B. Maleki and S. Ghanei, “A multi-objective optimization problem for multi-state series-parallel systems: A two-stage flow-shop manufacturing system,” Reliability Engineering and System Safety, vol. 136, pp. 62-74, Apr 2015. Article (CrossRef Link)

[27] Jafary B. and L. Fiondella, "A universal generating function-based multi-state system performance model subject to correlated failures,” Reliability Engineering and System Safety, vol. 152, pp. 16-27, Apr 2016. Article (CrossRef Link)

[28] EIMaraghy W., H.A. EIMaraghy, T. Tomiyama and L. Monostori, “Complexity in engineering design and manufacturing,” CIRP Annuals - Manufacturing Technology, vol. 61, pp. 793-814, Dec 2012. Article (CrossRef Link)

[29] Efthymiou K., D. Mourtzis, A. Pagoropoulos, N. Papakostas and G. Chryssolouris, "Manufacturing systems complexity analysis methods review," International Journal of Computer Integrated Manufacturing, vol. 29, no. 9, pp. 1025-1044, Jan 2015. Article (CrossRef Link)

[30] Alkan B., D.A. Vera, M. Ahmad, B. Ahmad and R. Harrison, "Complexity in manufacturing systems and its measures: a literature review,” European Journal of Industrial Engineering, vol. 12, no. 1, pp. 116-150, Jan 2018. Article (CrossRef Link)

[31] Frizelle G. and E. Woodcock, "Measuring complexity as an aid to developing operational strategy," International Journal of Operations and Production Management, vol. 15, no. 5, pp. 26-39, May 1995. Article (CrossRef Link)

[32] Frizelle G. and Y.M. Suhov, "An entropic measurement of queuing behavior in a class of manufacturing operations," the Royal Society of London Series A-Mathematical Physical and Engineering Sciences, vol. 457, pp. 1579-1601, Jul 2001. Article (CrossRef Link) 
[33] Efstathiou J., A. Calinescu and G. Blackburn. "A web-based expert system to assess the complexity of manufacturing organizations," Robotics and Computer Integrated Manufacturing, vol. 18, no. 3, pp. 305-311, Jun 2002. Article (CrossRef Link)

[34] EIMaraghy H.A., O. Kuzgunkaya and R.J. Urbanic, "Manufacturing systems configuration complexity,” CIRP Annals-Manufacturing Technology, vol. 54, no. 1, pp. 445-450, Jan 2005. Article (CrossRef Link)

[35] Kuzgunkaya O. and H.A. EIMaraghy, "Assessing the structural complexity of manufacturing systems configurations," International Journal of Flexible Manufacturing systems, vol. 18, no. 2, pp. 145-171, Jun 2006. Article (CrossRef Link)

[36] ElMaraghy H.A., T. AlGeddawy and S.N. Samy, "A model for assessing the layout structural complexity of manufacturing systems," Journal of Manufacturing Systems, vol. 33, no. 1, pp. 51-64, Jan 2013. Article (CrossRef Link)

[37] Samy S.N., T. AlGeddawy and H.A. ElMaraghy, "A granularity model for balancing the structural complexity of manufacturing systems equipment and layout," Journal of Manufacturing Systems, vol. 36, pp. 7-19, Jul 2015. Article (CrossRef Link)

[38] Zhu X.W., S.J. Hu, Y. Koren and S.P. Marin, "Sequence planning to minimize complexity in mixed-model assembly lines," in Proc. of 2007 IEEE International Symposium on Assembly and Manufacturing, pp. 251-258, Aug 2007. Article (CrossRef Link)

[39] Hu S.J., X. Zhu, H. Wang and Y. Koren, "Product variety and manufacturing complexity in assembly systems and supply chains," CIRP Annals- Manufacturing Technology, vol. 57, no. 1, pp. 45-48, Dec 2008. Article (CrossRef Link)

[40] Wang H. and S.J. Hu, "Manufacturing complexity in assembly systems with hybrid configurations and its impact on throughout," CIRP Annals-Manufacturing Technology, vol. 59, no. 1, pp. 53-56, Dec 2010. Article (CrossRef Link)

[41] Wang H., H. Wang and S.J. Hu, "Utilizing variant differentiation to mitigate manufacturing complexity in mixed-model assembly systems," Journal of Manufacturing Systems, vol. 32, no. 4, pp. 731-740, Oct 2013. Article (CrossRef Link)

[42] Rok V. and B. Peter, "Assessing operational complexity of manufacturing systems based on statistical complexity," International Journal of Production Research, vol. 50, no. 14, pp. 3673-3685, Jul 2012. Article (CrossRef Link)

[43] Smart J., A. Calinescu and L.H. Huatuco, "Extending the information-theoretic measures of the dynamic complexity of manufacturing systems," International Journal of Production Research, vol. 51, no. 2, pp. 362-379, Jan 2011. Article (CrossRef Link)

[44] Gershwin S.B. and J.E. Schor, "Efficient algorithms for buffer space allocation," Annals of Operations Research, vol. 93, pp. 117-144, Mar 2000. Article (CrossRef Link)

[45] Radhoui M. N. Rezg and A. Chelbi, "Integrated model of preventive maintenance, quality control and buffer sizing for unreliable and imperfect production systems," International Journal of Production Research, vol. 47, no. 2, pp. 389-402, Jan 2009. Article (CrossRef Link)

[46] Walid A.K., G. Ozhand and B. Fazle, "A nonlinear model for optimizing the performance of a multi-product production line," International Transactions in Operational Research, vol. 18, no. 5, pp. 561-577, May 2011.Article (CrossRef Link)

[47] Matta A., M. Pezzoni and Q. Semeraro, "A Kriging-based algorithm to optimize production systems approximated by analytical models," Journal of Intelligent Manufacturing, vol. 23, pp. 587-597, Jun 2012. Article (CrossRef Link)

[48] Papadopoulos C.T., M.E.J. O'Kelly and A.K. Tsadiras, "A DSS for the buffer allocation of production lines based on a comparative evaluation of a set of search algorithms," International Journal of Production Research, vol. 51, no. 14, pp. 4175-4199, Jul 2013. Article (CrossRef Link)

[49] Alfieri A., A. Matta and E. Pastore. "The time buffer approximated Buffer Allocation Problem: A row-column generation approach," Computers and Operations Research, vol. 115, pp. 1-14, Oct 2019. Article (CrossRef Link) 
[50] Renna P., "Adaptive policy of buffer allocation and preventive maintenance actions in unreliable production lines,” Journal of Industrial Engineering International, vol. 15, pp. 411-421, 2019. Article (CrossRef Link)

[51] Motlagh M. M., P. Azimi, M. Amiri and G. Madraki, "An efficient simulation optimization methodology to solve a multi-objective problem in unreliable unbalanced production lines," Expert Systems with Applications, vol. 138, pp. 1-22, Jul 2019. Article (CrossRef Link)

[52] Park T., "A two-phase heuristic algorithm for determining buffer sizes of production lines," International Journal of Production Research, vol. 31, no. 3, pp. 613-631, Mar 1993. Article (CrossRef Link)

[53] Demir L., S. Tunali and D. T. Eliiyi, "The state of the art on buffer allocation problem: a comprehensive survey,” Journal of Intelligent Manufacturing, vol. 25, pp. 371-392, May 2014. Article (CrossRef Link)

[54] Weiss S., J. A. Schwarz and R. Stolletz, "The buffer allocation problem in production lines: Formulations, solution methods, and instances,” IIE Transactions, vol 51, no. 5, pp. 456-485, Feb 2018. Article (CrossRef Link)

[55] Shu S. G., "Reliability analysis of computer integrated manufacturing system with buffers," Acta Automatica Sinica, vol. 18, no. 1, pp. 15-20.

[56] Zhu C., Liang X.M. and Yan D.H., "The Mechanism Research of a Novel Genetic Algorithm Based Species Selection,” in Proc. of International Conference on Computer Science and Software Engineering, pp. 462-466, Jun 2008. Article (CrossRef Link)

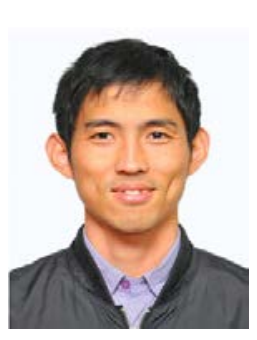

Jianguo Duan received his Ph.D. degree in Mechanical Manufacturing and Automation from Tongji University, China, in 2013. He is currently a professor at China Institute of FTZ Supply Chain, Shanghai Maritime University, China. His main research interests include modeling and simulation of discrete manufacturing system and digital-twin driven smart manufacturing.

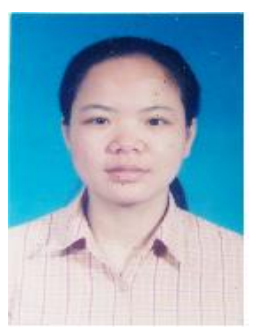

Nan Xie received her Ph.D. degree in Mechanical Manufacturing and Automation from Tongji University, China, in 2006. She is currently a professor at Sino-German College of Applied Sciences, Tongji University, Shanghai, China. Her main research interests include modeling and DES modeling and digital-twin driven smart manufacturing.

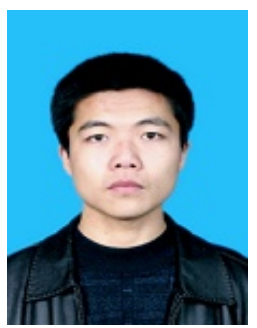

Lianhui Li received his Ph.D. degree in Aeronautics and Astronautics Manufacturing Engineering from Northwestern Polytechnical University, China, in 2016. He is currently a lecturer at North Minzu University, China. His current research interests include $\mathrm{CAD} / \mathrm{CAM}$ and logistics engineering. 